\title{
Combatendo as DCNT: uma abordagem diferente é necessária*
}

Tackling NCDs: a different approach is needed

Confrontando las ENT: es necesario un enfoque diferente

Jan De Maeseneer ${ }^{1 \star \star}$, Richard G Roberts² ${ }^{2}$ Marcelo Demarzo ${ }^{3}$, lona Heath ${ }^{4}$, Nelson Sewankambo ${ }^{5}$, Michael R Kidd ${ }^{6}$, Chris van Weel ${ }^{7}$, David Egilman ${ }^{8}$, Charles Boelen ${ }^{9}$, Sara Willems ${ }^{10}$

A Aliança DCNT (NCD Alliance) ${ }^{1}$ visa colocar as doenças crônicas não-transmissíveis (DCNT) na agenda global de saúde, a fim de enfrentar a chamada "crise das DCNT". Melhorar os indicadores de morbidade e mortalidade em 2015 dependerá, em grande medida, da prevenção e do controle das DCNT, especialmente nos países em desenvolvimento ${ }^{2}$.

Chamar a atenção mundial sobre as DCNT é oportuno, mas a Aliança DCNT parece oferecer uma estratégia conflitante para tal. Por um lado, uma abordagem vertical e orientada à doença é recomendada, por exemplo, propondo o desenvolvimento de uma combinação "multidroga" para pessoas com risco aumentado à doença cardiovascular. Por outro lado, a Aliança apela ao fortalecimento dos sistemas de saúde, com foco na Atenção Primária à Saúde (APS). No entanto, sua visão da APS é limitada e ambígua. Esta é vista como uma oportunidade para encontrar casos (para os programas orientados à doença), mas é ignorada como fonte de atenção integral, que integra e coordena cuidados para os problemas de saúde, envolvendo indivíduos, famílias e comunidade. É aqui que se encontra seu verdadeiro valor agregado para o cuidado em saúde e para a saúde das pessoas ${ }^{3}$.

Embora muito tenha sido aprendido com os programas verticais e orientados à doença, as evidências científicas sugerem que melhores resultados ocorrem quando se enfrenta as doenças por meio de uma abordagem integrada em um sistema de APS forte. Um exemplo é o Brasil, em que a cobertura terapêutica atinge quase $100 \%$, maior do que em países com APS menos robusta ${ }^{4}$. Os programas verticais orientados para HIV-AIDS, malária, tuberculose e outras doenças infecciosas criam duplicidade, uso ineficiente de recursos, lacunas no cuidado de pacientes com múltiplas comorbidades e reduzem a capacidade dos governos ao empurrar os melhores profissionais para fora do setor de saúde pública, concentrando-os no cuidado voltado a uma única doença ${ }^{5,6}$. Além disso, programas verticais causam desigualdade aos pacientes que não têm a doença 'certa', e drenagem interna dos melhores 'cérebros' entre profissionais de saúde?

\footnotetext{
Faculty of Medicine and Health Sciences; Department of Family Medicine and Primary Health Care, Ghent University, Ghent, Belgium.

jan.demaeseneer@ugent.be.

${ }^{2}$ Department of Family Medicine, University of Wisconsin School of Medicine and Public Health, Madison, WI, USA.

${ }^{3}$ Department of Preventive Medicine, Federal University of São Paulo, São Paulo, Brazil.

${ }^{4}$ Royal College of General Practitioners, London, UK.

${ }^{5}$ Makerere University College of Health Sciences, Kampala, Uganda.

${ }^{6}$ Faculty of Health Sciences, Flinders University, Adelaide, Australia.

${ }^{7}$ Department of Primary and Community Care, Radboud University Nijmegen Medical Centre, Nijmegen, Netherlands.

${ }^{8}$ Department of Family Medicine, Brown University, Providence, RI, USA.

${ }^{9}$ Secretariat of Global Consensus for Social Accountability of Medical Schools, Sciez-sur-Léman, France.

${ }^{10}$ Department of Family Medicine and Primary Health Care, Ghent University, Ghent, Belgium.

${ }^{*}$ Reprinted with permission from Elsevier (The Lancet, 2011, 6 September 2011, online first) - Reproducão autorizada do original em inglês, publicado no periódico The Lancet, detentor dos copyrights:

Maesenner JD, Roberts RG, Demarzo M, Heath I, Sewankambo N, Kidd MR, et al. Tackling NCDs: a different approach is needed. Lancet. 2011; [cited 2011 Sept 06]. Available at: http://dx.doi.org/10.1016/S0140-6736(11)61135-5

Conflito de interesse: declararam não haver.

${ }^{* \star}$ Autor correspondente
} 
As lições aprendidas de uma abordagem vertical e orientada para doenças infecciosas e tropicais negligenciadas ${ }^{8}$ deveriam nos inspirar a repensar a estratégia para as DCNT.

Em 2009, a Resolução WHA62.12, da Assembleia Mundial da Saúde, instigou os Estados membros "para incentivarem que programas verticais, incluindo aqueles para doenças específicas, sejam desenvolvidos, integrados e implementados no contexto da APS" ${ }^{\prime}$. Uma APS horizontal fornece oportunidade de integração de cuidados, enfrentando o problema da iniquidade em saúde, ao permitir que o foco nas DCNT ocorra concomitante ao acesso a outros problemas de saúde; dessa forma, evitando "a iniquidade pela doença" ${ }^{10}$.

Descrever o aumento da prevalência das DCNT como uma crise fornece material para um 'bom drama', mas pode levar a pensar que este problema é passível de uma solução rápida. As DCNT representam um conjunto de condições crônicas que requeretão esforços sustentados por muitas décadas. Assim, a solução seletiva e focal perseguida no combate às doenças infecciosas deve dar lugar a uma estratégia abrangente e duradoura, que afete e reflita a estrutura e a pesquisa nos serviços e sistemas de saúde.

Uma APS integrada e integradora é essencial para o combate às DCNT. As condições crônicas, muito mais do que as doenças infecciosas, são influenciadas pelas percepções e pelo comportamento dos pacientes. O manejo efetivo das DCNT vai exigir uma mudança do modelo orientado por problema para um orientado por objetivos e metas ${ }^{11}$. A gestão e o manejo a longo prazo das condições crônicas requer mais do que "o acesso aos medicamentos essenciais na APS” ${ }^{1}$. É necessário o empoderamento dos pacientes, a redução das barreiras para estilos de vida saudáveis e cuidados que reflitam os valores pessoais de cada paciente. Há evidências consistentes da efetividade da APS na redução das internações hospitalares relacionadas às DCNT, e a presença de morbidade múltipla entre aqueles com DCNT tem sido mais bem abordada na APS $^{12}$.

Não é suficiente apenas exortar os formuladores de políticas e os profissionais de saúde para promoverem sinergias entre os programas existentes para as DCNT e outras prioridades da saúde global. Deve-se fundamentalmente repensar a forma de abordar a complexidade dos problemas de saúde, tanto em países desenvolvidos quanto naqueles em desenvolvimento. Isso exigirá que as pessoas e seus valores sejam colocados no centro do processo, ao invés de doenças específicas.

A melhor resposta ${ }^{3}$ ao desafio das DCNT é promover cuidados centrados nas pessoas, por meio de investimentos numa APS integrada e fortalecida ${ }^{13}$, incluindo um número suficiente de profissionais bem treinados para tal modelo de atenção ${ }^{14}$. Pelo menos $50 \%$ dos profissionais de saúde formados deveriam ser treinados e capacitados aos cuidados de saúde primários. A Aliança DCNT calcula que um compromisso global de cerca de 9 bilhões de dólares americanos por ano será necessário para financiar as intervenções prioritárias. Nosso conselho é para que se acrescente outros 9 bilhões para fortalecer os serviços de APS locais nos mesmos países. Como resultado, milhões de pessoas poderão ter acesso a cuidados primários de saúde abrangentes, custoefetivos, de qualidade e que atendam às condições, incluindo as doenças infecciosas e as DCNT.

Estamos num momento importante de reflexão e devemos aprender com os erros passados, eventualmente bem intencionados. É tempo para responder aos objetivos da Resolução WHA62.12 e colocá-los em prática. Convidamos aos interessados para participarem nesta reflexão fundamental, e para tecer suas expertises numa ampla tapeçaria de cuidados e pesquisas mais eficazes e relevantes em saúde.

\section{Referências}

1. Beaglehole R, Bonita R, Horton R, Adams C, Asaria GP, Baugh V, et al. Priority actions for the non-communicable diseases crisis. Lancet. 2011;377:1438-47.

2. Stuckler D, Basa S, McKee ML. Drivers of inequality in Millennium Development Goal progress: a statistical analysis. PLoSMed. 2010;7:e1000241.

3. WHO. World Health Report Primary Health Care (Now More Than Ever). 2008. [accessed 2011 July 7]. Available at: http://www.who.int/whr/2008/en/index.html.

4. Barreto ML, Teixeira MG, Bastos FI, Ximenes RAA, Barata RB, Rodrigues LC. Successes and failures in the control of infectious diseases in Brazil: social and environmental context, policies, interventions, and research needs. Lancet. 2011;377:1877-89.

5. The Lancet. A strategic revolution in HIV and global health. Lancet. 2011;377:2055.

6. De Maeseneer J, van Weel C, Egilman D, Mfenyana K, Kaufman A Sewankambo N, et al. Funding for primary health care in developing countries: money from disease specific projects could be used to strengthen primary care. BMJ. 2008;336:518-9

7. Unger JP, De Paepe P, Green A. A code of best practice for disease control programmes to avoid damaging health care services in developing countries. Int J Health Plann Manage. 2003;18:S27-39.

8. Cavalli A, Bamba SI, Traore MN, Boelaert M, Coulibaly Y, Polman K, et al. Interactions between global health initiatives and country health systems: the case of neglected tropical diseases control program in Mali. PLoS Negl Trop Dis. 2010;4(8):e798.

9. Sixty-Second World Health Assembly. Resolution WHA62.12. Primary health care, including health system strengthening. May 22, 2009. [accessed 2011 July 7]. Available at: http://www.who.int/hrh/resources/A62_12_EN.pdf.

10. Starfield B. The hidden inequity in health care. Int $J$ Eq Health 2011;10:15.

11. Mold JW, Blake GH, Becker LA, et al. Goal-oriented medical care. Fam Med. 1991;23:46-51.

12. Starfield B. Challenges to primary care from co- and multi-morbidity. Prim Health Care Res Dev. 2011;12(1):1-2.

13. van Weel C, De Maeseneer J, Roberts R. Integrating personal and community health care. Lancet. 2008;372(9642):871-2.

14. Global Consensus for Social Accountability of Medical Schools. 2011. [accessed 2011 July 19]. Available at: http://www.healthsocialaccountability.org. 